\title{
Research Paper: Effectiveness of Joint Stiffness and Power Alternations in Different Shoe Insole Hard- ness on Injury Prevention During Jump-landing
}

\author{
Fatemeh Alirezaei Noghondar ${ }^{12^{*}} \mathrm{Q}$, Hamideh Khodaveisi ${ }^{2} \mathrm{Q}$
}

1. Department of Physical Education and Sport Sciences, Faculty of Sports Sciences, Ferdowsi University of Mashhad, Mashhad, Iran 2. Department of Physical Education, Faculty of Human Sciences, Hamedan Branch, Islamic Azad University, Hamedan, Iran.

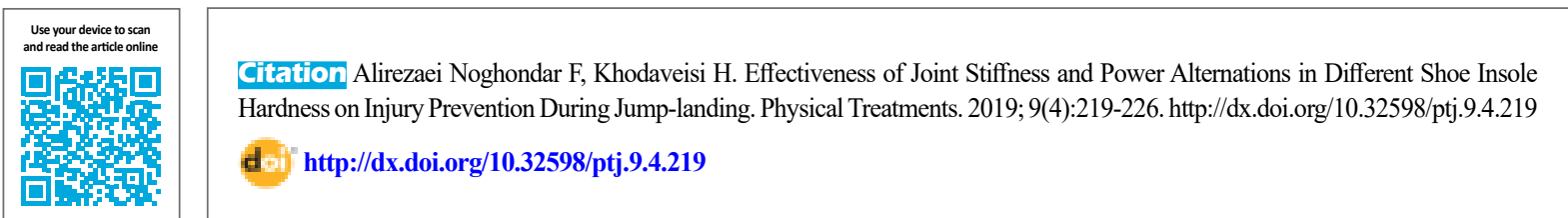

\section{(a) 0 (3)}

Article info:

Received: 03 Apr 2019

Accepted: 25 Aug 2019

Available Online: 01 Oct 2019

Keywords

Joint stiffness, Joint power, Insole, Jumplanding, Injury prevention

\section{A B S T RA C T}

Purpose: Studies on how the shoe insole hardness regulate joint stiffness and transfer energy in the lower extremity during jump landing are scarce. The current study aimed to determine the effectiveness of shoe insole changes in joint power and stiffness during landing from jumps.

Methods: Fifteen healthy male athletes volunteered to perform jump-landing in various shoe insole conditions. Kinematics and kinetics data were examined to calculate joint stiffness and peak negative power (absorbing power).

Results: The shoe insole hardness significantly affected ankle joint stiffness $(\mathrm{P} \leq 0.05)$. Furthermore, enhanced shoe inserts hardness increased ankle joint stiffness. Moreover, soft insole significantly increased negative peak power, compared to the hard insole condition $(\mathrm{P}=0.01)$.

Conclusion: Increasing the shoe insert hardness can higher affect the distal ankle joint, compared to the proximal joints. However, landing with soft shoe insoles increased the negative joint power during landing; it implied neuromuscular adaptation in response to the impact signals and elevated eccentric force by the knee extensors.

\section{* Corresponding Author:}

Fatemeh Alirezaei Noghondar, PhD.

Address: Department of Physical Education and Sport Sciences, Faculty of Sports Sciences, Ferdowsi University of Mashhad, Mashhad, Iran.

Phone: +98 (51) 38805419

E-mail:alirezaee@um.ac.ir 


\section{Highlights}

- The obtained results suggested changes in the joint ankle stiffness in hard insole condition, compared with other conditions, as a neuromuscular adaptation.

- Wearing a shoe with soft insole condition can result in greater negative knee joint power during landing, compared to hard and medium insoles.

\section{Plain Language Summary}

A neuromuscular adaptation occurs in response to the hard insole condition with increasing the ankle joint stiffness. However, negative knee joint power was greater with soft insole conditions during jump-landing. The more ankle joint stiffness may increase the decelerated mass at landing which implies an increased risk of landing injuries with hard insole. A greater negative knee joint power during landing when using soft insole is an indication of the eccentric force on knee extensor muscles to attenuate loading impact.

\section{Introduction}

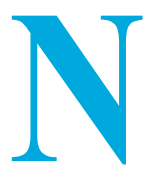

umerous individuals use shoe inserts for athletic and physical activities, including running and jump-landing, to reduce sports injuries [1]. Lower extremity experiences a large impact force of about 3 to 7 times of weight during landing [2-3]. Previous investigations reported a relationship between the cushioning properties of footwear and its implication in injury prevention [4].

Current biomechanical investigations have focused on the cushioning characteristics of athletic footwear. These studies have reported debating results on whether softer shoe insert materials alter Ground Reaction Force (GRF) characteristics. It is believed that softer midsole can reduce impact force and loading during running, compared to harder ones [5]. However, in some cases, wearing shoes with different insole hardness do not affect impact force characteristics during landing and lower extremity joint angle [6]. Meanwhile, some researchers have demonstrated no significant relationship between insole densities and vertical reaction force in running [4, 7-8]. However, the literature data are limited in this respect.

Previous research has identified that subjects modify their landing mechanics when landing with different shoe cushioning or surface; this occurs by making their ankle joint stiffer while the shoe or surface become more compliant [9]. For example, joint stiffness increased by softer shoe cushioning during running [10]. These results focused on the role that joint stiffness plays in locomotion, as well as function [11]. Furthermore, the interaction between insole hardness and joint stiffness in response to the different shoe conditions is important. It should be considered as a measure related to the lower extremity injuries and mechanical loads transmitted to the musculoskeletal system in response to the environmental changes [12]. Surprisingly, a more flexible joint could greater reduce the load transmitted through the joint, compared to a stiffer joint [12].

Additionally, lower extremity joint power represents critical estimates of joint energy during landing. DeVita and Skelly (1992) explored the effect of a stiff double-leg landing on reducing joint power and eccentric force [13]. However, the results of studies regarding are controversial on the lower extremity joints responses to the different shoe insole densities during landing. Therefore, the function of lower extremity joints could be tuned during jump-landing with different shoe insole hardness to accommodate the impact force at foot strike. The primary hypothesis was that the lower extremity joints would display a different behavior in response to changing the shoe insole hardness during jump-landing.

\section{Materials and Methods}

A sample of 15 physically active participants (Mean \pm SD age: $21.33 \pm 2.42$ years; height: $180.5 \pm 10.00$ $\mathrm{cm}$; weight: $69.08 \pm 7.67 \mathrm{~kg}$ ) were recruited for the investigation. the inclusion criteria were being physically active for at least 30 minutes and 3 times per week, of the lack of Anterior Cruciate Ligament (ACL) injuries, neurologic disorders, and lower extremity injuries in the $\geq 6$ months before data collection. All study participants provided written informed consent. Furthermore, the 
study procedures were approved by the University Institutional Review Board. An eight-camera Oqus system (Qualisys, Inc., Gothenburg, Sweden) with the sampling frequency of $120 \mathrm{~Hz}$ was used to capture three dimensional lower extremities motions. Additionally, these motions were tracked using the Qualisys Track Manager software (Qualisys, Inc., Gothenburg, Sweden). To collect ground reaction force, a force platform (type 4060$\mathrm{NC}$; AMTI Corporation, Columbus, $\mathrm{OH}$ ) was located in the center of the capture volume with a sampling frequency of $1000 \mathrm{~Hz}$.

The study participants' height and weight were recorded prior to data collection. All study participants were requested to perform two tasks, separately; jump-landing and Maximum Vertical Jump (MVJ) trials while wearing the same sports shoes (Nike Air Max Glide sneakers). The trials were conducted in 3 different shoe insole constructs of polyurethane foam with different hardness characteristics according to the following manufacturing tests: Asker insole C-40 (soft), Asker C-52 (medium), Asker C-65 (hard). Retro-reflexive markers were placed onto the skin in the right lower extremity and pelvic landmarks of subjects, based on previous research studies [14].

To perform double-legged jump landings, the study participants were instructed based on previous investigations. The double-legged jump consisted of jumping forward and vertically on the force plate to hit a suspended ball which was set as $50 \%$ of MVJ. The final task comprised landing with both feet on force-plate with desired techniques and subsequently performing maximum vertical jump [15]. For each participant, 3 practice trials and 5 successful testing trials with a 30 -second rest between trials were performed to prevent the effects of fatigue. Trials were considered valid if the study subjects landed with each foot completely contacting the force plate, and performed and the subsequent maximal vertical jump.

Row data were exported and further processed using MATLAB program (v. 2015b, Math works; Natick,
USA). Three dimensional coordination data were filtered using a low-pass, fourth-order, zero-phase-lag Butterworth filter with a cutoff frequency of $10 \mathrm{~Hz}$; this was applied to calculate joint angular positions and instantaneous joint angular velocities [16]. The GRF data were filtered at $60 \mathrm{~Hz}$ with a low-pass, fourth-order, zerophase-lag Butterworth filter [17]. The inverse dynamic approaches were used to combine the kinematic and anthropometric data. This process helped to calculate the net internal joint moments of force and the net internal force in lower extremity joints and segments during landing [18]. All variables were extracted from initial contact to take-off and resampled at $1 \%$ increments $(\mathrm{N}=101)$. The initial contact occurred at the first instant GRF that exceeded $10 \mathrm{~N}$ and take off occurred at the first instant GRF that reached to $<10 \mathrm{~N}$. The ratio of the change in the joint moment $(\Delta \mathrm{M})$ to the joint angular displacement $(\Delta \theta)$ was expressed as the joint stiffness [19] (Figure 1). The product of joint angular velocity and moment normalized to the body weight [20] (Watt $\times$ BW-) were calculated as the joint power (Figure 2). Before conducting the statistical analysis, all measures were averaged for each study participant within trials in each experimental condition. A repeated measures Analysis of Variance (ANOVA) was used to determine the influence of shoe insole hardness on the ankle and knee joint stiffness at power alpha level of 0.05 . Bonferroni posthoc test was used to compare the differences between the two conditions. The Effect Size (ES) was calculated to further determining the mean differences.

\section{Results}

The ankle joint stiffness was significantly influenced by shoe insert condition $(\mathrm{F}=3.12, \mathrm{P}=0.05, \mathrm{ES}=0.5)$ (Table 1). Post-hoc analysis indicated that ankle joint stiffness significantly differed in pair-wise comparisons $(\mathrm{P}=0.04)$. Pair-wise comparisons revealed that the ankle joint stiffness was greater as shoe insole hardness increased, compared to soft insole condition. The obtained results confirmed that knee and hip joint stiffness were not significantly influenced by shoe insole conditions $(F=0.18$,

Table 1. The joint stiffness values (Nm/degree) for hard, soft, and medium insoles during jump-landing task

\begin{tabular}{|c|c|c|c|c|c|}
\hline \multirow{2}{*}{ Variables } & \multicolumn{3}{|c|}{ Mean $\pm S D$} & \multirow{2}{*}{$\mathbf{F}$} & \multirow{2}{*}{$\mathbf{P}$} \\
\hline & Hard & Medium & Soft & & \\
\hline Ankle joint stiffness & $1.27 \pm 1.00$ & $0.75 \pm 0.45$ & $0.74 \pm 0.50$ & 3.12 & 0.05 \\
\hline Knee joint stiffness & $1.16 \pm 0.70$ & $0.90 \pm 0.75$ & $1.18 \pm 1.00$ & 0.18 & 0.88 \\
\hline Hip joint stiffness & $1.98 \pm 0.43$ & $0.91 \pm 0.70$ & $1.5 \pm 0.85$ & 0.9 & 0.66 \\
\hline
\end{tabular}


Table 2. The joint power Values (Watt/kg) for hard, soft, and medium insoles during jump-landing task

\begin{tabular}{|c|c|c|c|c|c|}
\hline \multirow{2}{*}{ Variables } & \multicolumn{3}{|c|}{ Mean $\pm S D$} & \multirow{2}{*}{$\mathbf{F}$} & \multirow{2}{*}{$\mathbf{P}$} \\
\hline & Hard & Medium & Soft & & \\
\hline Ankle joint power & $31.50 \pm 13.19$ & $33.13 \pm 11.35$ & $36.61 \pm 8.20$ & 1.6 & 0.22 \\
\hline Knee joint power & $2.32 \pm 1.20$ & $4.87 \pm 3.80$ & $4.46 \pm 2.42$ & 10.6 & 0.002 \\
\hline Hip joint power & $24.00 \pm 6.45$ & $22.88 \pm 7.48$ & $22.54 \pm 6.71$ & 0.48 & 0.62 \\
\hline
\end{tabular}

$\mathrm{P}=0.83$; and $\mathrm{F}=0.9, \mathrm{P}=0.66$ ). Furthermore, the three different shoe condition significantly influenced knee joint power $(\mathrm{F}=10.6, \mathrm{P}=0.002$, $\mathrm{ES}=0.6$ ) (Table 2). Post-hoc comparisons suggested that peak negative power significantly increased in soft insole condition, compared to the hard condition during landing $(\mathrm{P}=0.01)$. There was no significant difference in negative ankle and hip joint power between shoe insole conditions $(\mathrm{F}=1.6, \mathrm{P}=0.22$; and $\mathrm{F}=0.48, \mathrm{P}=0.48$ ) (Table 2).

\section{Discussion}

The current study characterized the joint stiffness and power in the lower extremity during the jump-landing using different footwear insole densities. The obtained results revealed an increased joint ankle stiffness in hard insole condition. The joint stiffness is considered as the displacement occurring in joint relevant to the impact applied to the joint. Footwear and insoles may alter the sensory feedback received from the environment. These findings supported the previous research results, in which the ankle stiffness was greater in shod running condition, compared to barefoot running [21].
To modify joint locomotion, joint stiffness should be reduced as a result of 'bottoming out effect'. The possible interpretation for this effect may be the result of reaching the insole to the lowest in response to the ground contact. Therefore, individuals would have a better sense of contact and adjust their joint stiffness in response to the impact force. Joint stiffness represents a measure of the extent to which joint displacement occurs for a given moment. In other words, the ankle joint in soft insole condition is more compliant representing reduced joint stiffness to absorb more impacts during landing. A more compliant joint will attenuate the energy of the impact to a greater extent compared to a stiffer joint. As a result, in hard insole condition, sufficient sensory information may not provide for the ankle joint.

An essential finding was that the ankle joint was less stiff than the knee and hip joints in response to shoe insole density changes. The ankle joint is dominantly active during locomotion and ground contact; therefore, kinetic energy or ground reaction impact will be initially received at this joint during landing $[10,22]$. It means that plantar flexor muscles attenuate and reduce the ma-
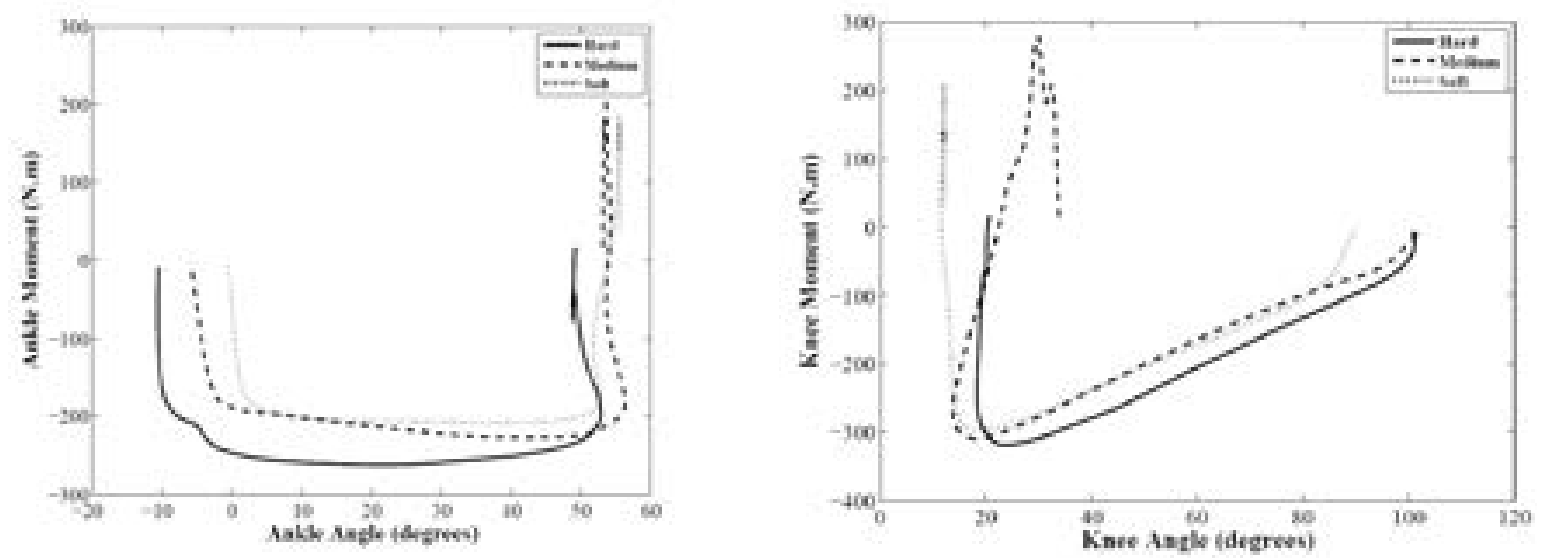

PHYSICAL TREA TMENTS

Figure 1. Representative profiles of a study subject for the ankle (top), knee (middle), and hip (bottom) joint stiffness during the landing phase

The landing phase was defined by the duration of initial contact to toe-off. 

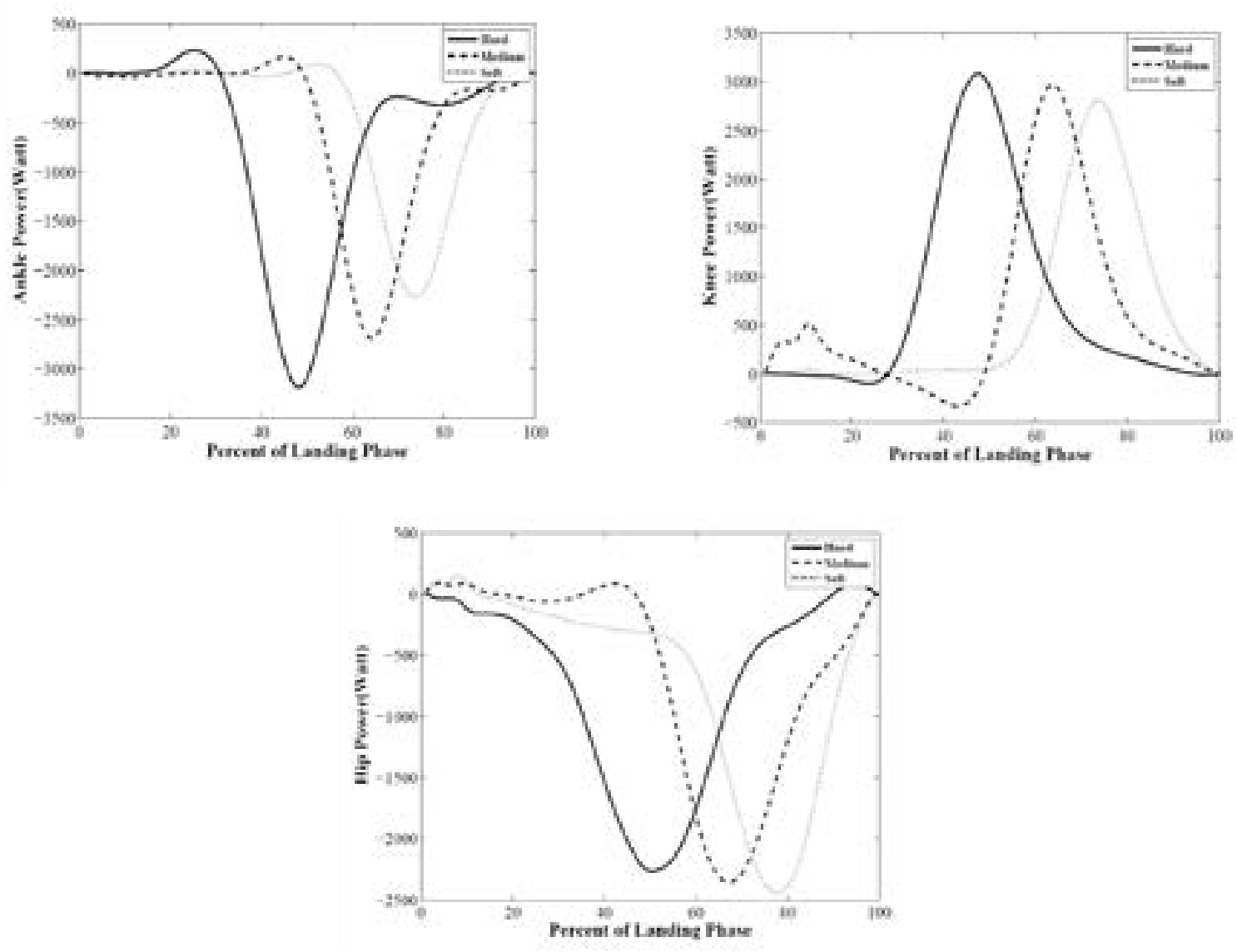

PHYSICAL TREA $\ M E N T S$

Figure 2. Representative profiles of a study subject for the ankle (top), knee (middle), and hip (bottom) joint power during the landing phase

The landing phase was defined by the duration of initial contact to toe-off.

jority of kinetic energy and impact force during dynamic tasks, like landing; this process limits energy transference within the kinetic chain [2].

An increased negative knee joint power was observed during landing in soft insole condition. Several studies reported increased knee joint power during landing; it represents the dominant role of knee extensor muscles in absorbing energy or eccentric force to attenuate impact [13, 23-24]. The knee extensor muscles might significantly affect energy absorbing during landing. Besides, the negative joint power was influenced by soft shoe insole conditions during landing. Such data revealed increased eccentric mechanical force on the knee extensor muscles to control joint locomotion and attenuate kinetic energy. To our knowledge, no previous data have been reported regarding the effect of shoe insole hardness on lower extremity joint stiffness and power during jumplanding; therefore, it is impossible to compare the present findings with other data.

\section{Conclusion}

The cushioning properties provided by shoe insole significantly affected the ankle joint stiffness; ankle joint stiffness increased in hard insole condition. This finding is interesting, because the ankle joint stiffness is clinically associated with injury risk. Increased ankle joint stiffness may increase the decelerated mass at landing. Moreover, it is associated with an increased risk of landing injuries; therefore, athletes should consider this potential increased injury risk when wearing shoes with a hard insole. The study results indicated a greater negative knee joint power during landing with soft insole condition, compared to the other conditions. However, the current investigation results may also have important implications for the loads imposed on the certain tissues of the lower extremity while landing with different shoe insole hardness. However, further work is necessary to better investigate the biomechanical responses to this type of shoe insole. 


\section{Ethical Considerations}

\section{Compliance with ethical guidelines}

All ethical principles were considered in this article and all of the participants completed the written consent form. Also, this study was confirmed by scientific board of sport science faculty of Ferdowsi University of Mashhad.

\section{Funding}

This work was supported by Ferdowsi University of Mashhad under grant number of 38868.

\section{Authors' contributions}

All authors contributed in designing, running, and writing all parts of the research.

\section{Conflict of interest}

The authors declared no conflict of interest.

\section{Acknowledgments}

The authors would like to thank the participants for their valuable cooperation.

\section{References}

[1] Brund R N, Nielen R, Parner E, Rasmussen S, Voigt M. Changes in the running-related injury incidence rate ratio in a 1000-km explorative prospective cohort study involving two unspecific shoe changes. Footwear Science. 2019; 11(2):63-70. [DOI:10.1080/19424280.2018.1529063]

[2] Gross TS, Nelson RC. The shock attenuation role of the ankle during landing from a vertical jump. Medicine \& Science in Sports \& Exercise. 1988; 20(5):506-14 [DOI:10.1249/00005768-198810000-00013]

[3] McNitt-Gray JL. Kinetics of the lower extremities during drop landings from three heights. Journal of Biomechanics. 1993; 26(9):1037-46. [DOI:10.1016/S0021-9290(05)80003-X]

[4] Clarke TE, Frederick EC, Cooper LB. Effects of shoe cushioning upon ground reaction forces in running. International Journal of Sports Medicine. 1983; 4(4):247-51. [DOI:10.1055/s-2008-1026043] [PMID]

[5] Lafortune MA, Henning EM. Dominant role of interface over knee angle for cushioning impact loading and regulating initial leg stiffness. Journal of Biomechanics. 1996; 29(12):15239. [DOI:10.1016/S0021-9290(96)80003-0]

[6] Alirezaei NF, Bressel E. Effect of shoe insole density on impact characteristics and performance during a jump-landing task. Footwear Science. 2017; 9(2):95-101. [DOI:10.1080/1942 4280.2017.1305003]

[7] Hamill J, Russell E, Gruber A, Miller R. Impact characteristics in shod and barefoot running. Footwear Science. 2011; 3(1):33-40. [DOI:10.1080/19424280.2010.542187]

[8] Zhang S, Clowers K, Kohstall C, Yu YJ. Effects of various midsole densities of basketball shoes on impact attenuation during landing activities. Journal of Applied Biomechanics. 2005; 21(1):3-17. [DOI:10.1123/jab.21.1.3] [PMID]

[9] Bishop M, Fiolkowski P, Conrad B, Brunt D, Horodyski M. Athletic footwear, leg stiffness and running kinematics. Journal of Athletic Training. 2006; 41(4):376-92. [PMCID] [PMID]

[10] Kulmala JP, Kosonen J, Nurminen J, Avela J. Running in highly cushioned shoes increases leg stiffness and amplifies impact loading. Scientific Reports. 2018; 8:17496. [DOI:10.1038/s41598-018-35980-6] [PMID] [PMCID]

[11] Denoth J. Load and the locomotor system and modeling. In: Nigg B, editor. Biomechanics of Running Shoes. Champagne: Human Kinetics Publishers; 1986.

[12] Hamill J, Gruber AH, Derrick TR. Lower extremity joint stiffness characteristics during running with different footfall patterns. European Journal of Sport Science. 2014; 14(2):130-6. [DOI:10.1080/17461391.2012.728249] [PMID]

[13] Devita P, Skelly WA. Effects of landing stiffness on join kinetics and energetics in the lower extremity. Medicine \& Science in Sports \& Exercise. 1992; 24(1):108-15 [DOI:10.1249/00005768-199201000-00018]

[14] McClay L, Manal K. Coupling parameters in runners with normal and excessive pronation. Journal of Applied Biomechanics. 1997; 13(1):109-24. [DOI:10.1123/jab.13.1.109]

[15] Debiaso J C, Russell MA, Butler RJ, Nunly JA, Queen RM Changes on plantar loading based on shoe type and sex during a jump-landing task. Journal of Athletic Training. 2013; 48(5):601-9. [DOI:10.4085/1062-6050-48.3.08] [PMID] [PMCID]

[16] Decker MJ, Torry MR, Wyland DJ, Sterett WI, Steadman RJ. Gender differences in lower extremity kinematics, kinetics and energy absorption during landing. Clinical biomechanics (Bristol, Avon). 2003; 18(7):662-9. [DOI:10.1016/S0268 0033(03)00090-1]

[17] Kulas AS, Schmitz RJ, Shultz SJ, Watson MA, Perrin DH Energy absorption as a predictor of leg impedance in highly trained females. Journal of Applied Biomechanics. 2006; 22(3):177-85. [DOI:10.1123/jab.22.3.177] [PMID]

[18] Gagnon D, Gagnon M. The influence of dynamic factors on triaxial net muscular moments at the L5/S1 joint during asymmetrical lifting and lowering. Journal of Biomechanics. 1992; 25(8):891-3. [DOI:10.1016/0021-9290(92)90229-T]

[19] Horita T, Komi PV, Nicol C, Kyrorlaeinen H. Interaction between pre-landing activities and stiffness regulation of the knee joint musculoskeletal system in the drop jump: Implications to performance. European Journal of Applied Physiology. 2002; 88:76-84. [DOI:10.1007/s00421-002-0673-6] [PMID]

[20] Yeow CH, Lee PV, Goh JC. Regression relationships of landing height with ground reaction forces, knee flexion angles, angular velocities and joint power during dou- 
ble-leg landing. Knee. 2009; 16(5):381-6. [DOI:10.1016/j. knee.2009.02.002] [PMID]

[21] Chambon N, Delattre N, Gueguen N, Berton E, Rao G. Is midsole thickness a key parameter for the running pattern? Gait Posture. 2014; 40(1):58-63. [DOI:10.1016/j.gaitpost.2014.02.005] [PMID]

[22] Farley CT, Gonzales O. Leg stiffness and stride frequency in human running. Journal of Biomechanics. 1996; 29(2):181-6. [DOI:10.1016/0021-9290(95)00029-1]

[23] Fukuda, H. Biomechanical analysis of landing on surfaces with different stiffness. In: DeGroot G, Hollander AP, Hiujing PA, van Ingen Schenau GJ (editors). Biomechanics XI-B. Amsterdam: Free University Press, 1988.

[24] Bobbert MF, Huijing PA, van Ingen Schenau. An estimation of power output and work done by the human triceps surae muscle-tendon complex in jumping. Journal of Biomechanics. 1986; 19(11):899-906. [DOI:10.1016/0021-9290(86)90185-5] 
This Page Intentionally Left Blank 